\title{
Die Dorn-Therapie - Volksheilkunde für eine Volkskrankheit
}

Fast $\mathbf{8 0 \%}$ der Erwachsenen haben im Laufe ihres Lebens ein- oder mehrmals länger andauernde Schmerzen „im Kreuz“. Rückenschmerzen stellen ein großes volksgesundheitliches Problem dar. Die Dorn-Methode, um 1975 entwickelt von dem Allgäuer Landwirt Dieter Dorn, ist eine „volksheilkundliche" Methode in der Tradition der Laienheiler. Dennoch fand sie aufgrund ihrer Einfachheit und Wirksamkeit rasche Verbreitung auch bei Heilpraktikern, Ärzten und Physiotherapeuten und ist heute eine anerkannte komplementärmedizinische manuelle Methode. Bei der Dorn-Therapie werden die Beinlängen korrigiert und falsch stehende Wirbel auf sanfte Weise wieder gerichtet.

\section{Eine Methode - viele Vorteile}

Die Dorn-Therapie ist eine effektive und sanfte manuelle Therapie, mit der sich Wirbelsäulen- und Gelenkblockaden rasch und dauerhaft beseitigen lassen. Als Verfahren, das ursprünglich aus der Volksmedizin kam, ist sie schnell erlernbar, sicher und hervorragend auch zur Krankheitsvorbeugung geeignet. Die Grundlagen werden normalerweise in einem Wochenendkurs vermittelt, nach dem Sie sofort mit dem selbstständigen Behandeln beginnen können. Dies ist möglich, weil für die Manipulationen - anders als bei z.B. der Chiropraktik - keine langen Hebel, keine Vorspannung und keine plötzlichen Bewegungen verwendet werden. Die Behandlung ist somit für den Patienten angenehmer und angstfreier; die Gelenke knacken und krachen weder während der Behandlung noch danach.

Die Behandlung selbst erfolgt in der Bewegung und immer diesseits der Schmerzgrenze des Patienten. Somit bringt die Methode ihr eigenes Sicher- heitsnetz mit. Außerdem lässt die Druckschmerzhaftigkeit der zu behandelnden Punkte sofort nach der Korrektur eines blockierten Wirbels oder Gelenks nach und ermöglicht eine sofortige Erfolgskontrolle.

Da bei der Dorn-Therapie grundsätzlich der gesamte Bewegungsapparat behandelt wird und nicht nur die Stellen, die gerade wehtun, ist sie ausgezeichnet zur Gesundheitspflege geeignet. Wirbelund Gelenkblockaden können nämlich lange Zeit existieren, ohne offensichtliche Symptome hervorzurufen. Irgendwann führen die falsche Belastung der Gelenke und die ungünstige Statik unweigerlich zu Gesundheitsproblemen. Das Beheben „stummer“ Blockaden entlastet den Körper von der Kompensationsarbeit und stellt die bislang dafür aufgewendete Energie für andere Aufgaben zur Verfügung. Mithilfe der Traditionellen Chinesischen Medizin (TCM) kann die allgemein kräftigende und aufbauende Wirkung der Dorn-Therapie erklärt werden: Die wiederholten Streichungen von unten nach oben entlang der gesamten Wirbelsäule tonisieren sanft das Lenkergefäß (Du Mai), den auf der Körperrückseite mittig verlaufenden Meridian, der zusammen mit dem Konzeptionsgefäß (Ren Mai) auf der Körpervorderseite das „Hauptstromkabel“ des Körpers darstellt.

\section{Was können wir erreichen?}

Die Hauptindikationen für die Dorn-Therapie sind akute und chronische Rückenschmerzen und andere Schmerzen des Bewegungsapparats, weil diese regelmäßig von Wirbel- und Gelenkblockaden verursacht werden. Blockaden der Brustund Halswirbelsäule allein können Kopfschmerzen, Schwindel und Herzrhythmusstörungen verursachen, und sie tragen wesentlich zur Pathogenese von Empfindungsstörungen in Armen und Händen, zu Epikondylitis und dem Karpaltunnelsyndrom bei. Darüber hinaus schränken Wirbelblockaden häufig die Impulsweiterleitung der Spinalnerven ein. Das Aufheben dieser Blockaden stellt die optimale Funktion des Nervensystems und somit die Steuerung für das reibungslose Funktionieren von inneren Organen und Abläufen im Körper wieder her. Deswegen sprechen auch innere Beschwerden häufig gut auf die Dorn-Therapie an. Patienten mit chronisch-degenerativen Erkrankungen wie Arthrosen, Morbus Bechterew oder Morbus Scheuermann erfahren durch die Dorn-Therapie häufig Schmerzlinderung, auch wenn das Krankheitsbild selbst nicht aufgehoben werden kann.

\section{Grenzen der Dorn-Therapie}

Die Dorn-Therapie darf nicht angewendet werden bei Frakturen, Tumoren und bei Patienten mit eingeschränkter Schmerzwahrnehmung oder Bewusstseinstrübung, z.B. durch Injektionen, Medikamente oder Alkohol. Die Behandlung von Schwangeren, Blutern, Patienten mit Gleitwirbeln, Bandscheibenvorfall oder Osteoporose darf nur sehr vorsichtig erfolgen und sollte erfahrenen Therapeuten vorbehalten bleiben, deren Behandlungsweise mehr auf Technik als auf Kraftaufwand beruht. Bei erstmalig auftretenden Rückenschmerzen ist im Rahmen der Sorgfaltspflicht vor der Behandlung eine vollständige schulmedizinische Diagnostik durchzuführen.

\section{Durchführung der Dorn-Therapie}

Der Ablauf einer Dorn-Behandlung erfolgt stets nach dem Motto „Das Haus von unten nach oben aufbauen“, das heißt: Zunächst werden die Beinlängen miteinander verglichen und eine eventuell bestehende Längendifferenz durch Aufheben von Becken-, Knie- und Knöchelblockaden ausgeglichen. Anschließend wer- 
den Blockaden der Iliosakralgelenke, der Lenden-, Brust- und Halswirbelsäule diagnostiziert und korrigiert, wobei Diagnose und Behandlung nahtlos ineinander übergehen. Zum Abschluss werden noch Schulter-, Ellbogen- und Handgelenke korrigiert und ggf. die Kiefergelenke. Darüber hinaus führen die Patienten über mehrere Wochen täglich eine oder mehrere Übungen aus, die - anders als bei Krankengymnastik - lediglich einige Sekunden in Anspruch nehmen. Durch die täglichen Impulse gewöhnt sich der Körper an die korrekten Gelenkstellungen, und der Behandlungserfolg bleibt dauerhaft stabil.

Die für die Behandlung notwendigen Hilfsmittel sind ein gutes Massageöl und ein Brettchen von ca. $5 \mathrm{~cm}$ Höhe (ersatzweise geht auch ein Telefonbuch).

\section{Behandlung im Stehen: LWS und untere BWS (Th 7 bis Th 12)}

Die Patientin steht mit leicht vorgebeugtem Oberkörper vor der Behandlungsliege und stützt sich mit beiden Händen auf der Liege ab ( Abb. 1). Ihre Beine stehen etwa hüftbreit auseinander. Der Therapeut steht hinter der Patientin und ölt den Rücken im Bereich der Wirbelsäule von L 5 bis Th 12 ein. Anschließend sucht er die Dornfortsätze des fünften Lendenwirbels und legt seine Daumen rechts und links daneben. Mit leichtem Druck gleitet er an der Wirbelsäule mehrfach neben den Dornfortsätzen bis zum siebten Brustwirbel nach oben und merkt sich, wo er seitliche Verschiebungen und Muskelverhärtungen um die Dornfortsätze findet. Wenn alle Wirbel „richtig“ stehen, gleiten die Daumen parallel nach oben wie in einer Schiene. Behandlungsbedürftige Wirbel machen sich durch Erhebungen auf dieser Schiene bemerkbar, durch die der Daumen zur Außenseite abrutscht. Meist sind diese Punkte auch druckdolent. Sind sie es nicht, ist der betreffende Wirbel nicht behandlungsbedürftig. Die Asymmetrie hat dann eine andere Ursache, z.B. einen unregelmäßig geformten Wirbel.

Die Behandlung wird an einem nach rechts verschobenen Wirbel erklärt: Der Therapeut steht rechts von der Patientin. Drei Wirbel unterhalb des zu behandelnden Wirbels legt er den Daumen der linken Hand rechts am Dornfortsatz an. Um

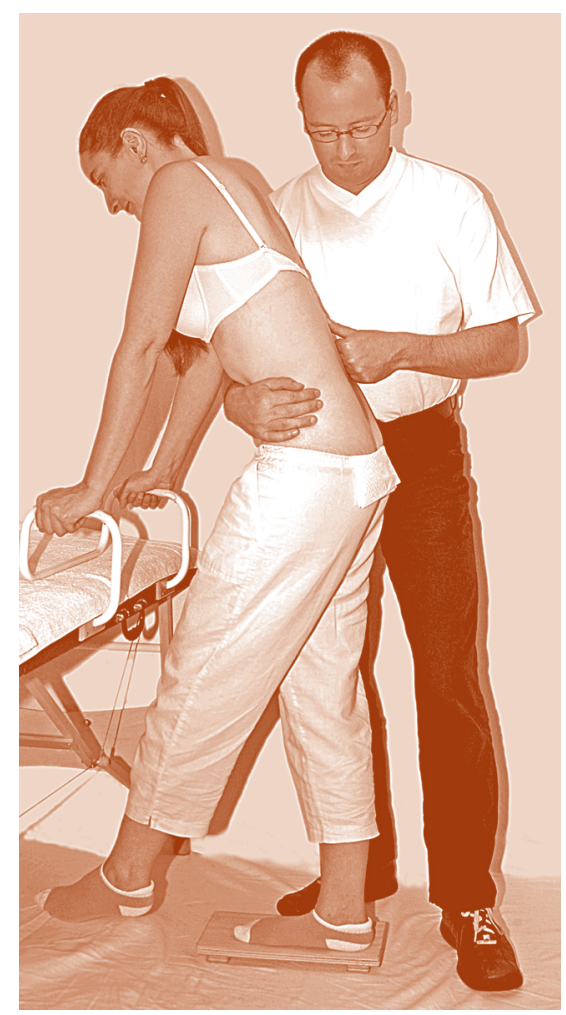

Abb. 1 Behandlung von Lendenwirbelsäule und unterer Brustwirbelsäule. Quelle: siehe [1]

Überlastung vorzubeugen, stützt er seinen Daumen auf ihrer Faust ab. Mit der rechten Hand umgreift er die Patientin an der Hüfte. Die Patientin stellt sich mit dem rechten Bein auf ein Brettchen und pendelt mit dem linken Bein vor und zurück. Der Therapeut kann nun mit dem Daumen eine leicht schwingende Bewegung spüren und gleitet von unten nach oben an der rechten Seite der Dornfortsätze entlang. An dem verschobenen Wirbel bleibt er etwa zwei Sekunden lang und erhöht den Druck leicht. Dann gleitet er weiter. Sollten mehrere Wirbel zur gleichen Seite verschoben sein, verweilt der Daumen auch an diesen Stellen wieder einige Zeit, bevor er weitergeht. Dieser Schritt ist drei- bis viermal zu wiederholen. Um eine Verkrampfung der Patientin zu vermeiden, sollte der Therapeut dann die Seite wechseln, auch wenn auf der anderen Seite kein Wirbel verschoben ist.
Hinweise für die Behandlung im Stehen

Auch wenn nur einzelne Wirbel verschoben sind, ist es sinnvoll, eine größere Strecke mit dem Daumen zu behandeln und nicht am schmerzhaftesten Punkt anzufangen. Der richtige Druck ist häufig ein wenig schmerzhaft, wird von der Patientin aber eher als lösend oder befreiend empfunden. Ausweichbewegungen sind eine Schutzreaktion, wenn die momentane Schmerzschwelle überschritten wird. Dann muss mit weniger Druck und häufigeren Wiederholungen behandelt werden. Bei nach links verschobenen Wirbeln pendelt die Patientin mit dem rechten Bein, während der Therapeut mit seiner rechten Hand von der linken Seite aus Richtung Mitte arbeitet.

\section{Behandlung im Sitzen: BWS (Th 1 bis T 9)}

Die Patientin sitzt mit leicht gebeugtem Oberkörper auf einem Hocker, ihre Arme können frei schwingen. Der Therapeut steht hinter der Patientin. Nachdem er den Bereich der oberen Brustwirbelsäule eingeölt hat, legt er die Daumen rechts und links neben den achten Brustwirbel und gleitet mehrfach an den Dornfortsätzen entlang nach oben bis zum siebten Halswirbel ( Abb. 2). Er merkt sich die Wirbel, an denen ein Daumen nach außen gleitet bzw. abgebremst wird. Die Behandlung entspricht der Behandlung der Lendenwirbelsäule mit dem Unterschied, dass die Patientin jetzt mit dem Arm pendelt und nicht mit dem der Muskelverhärtung gegenüber liegenden Bein.

Th 7 bis Th 9 können sowohl im Sitzen als auch im Stehen behandelt werden. Der Therapeut wählt die Variante, bei der er die Bewegung in der Wirbelsäule am besten spüren kann.

\section{Untersuchung der HWS im Sitzen}

Der Therapeut ölt den Hals der Patientin gut ein. Mit beiden Daumen gleitet er gleichzeitig von unten nach oben in der anatomischen Rille entlang. Dabei achtet er darauf, den Hals der Patientin nicht mit 


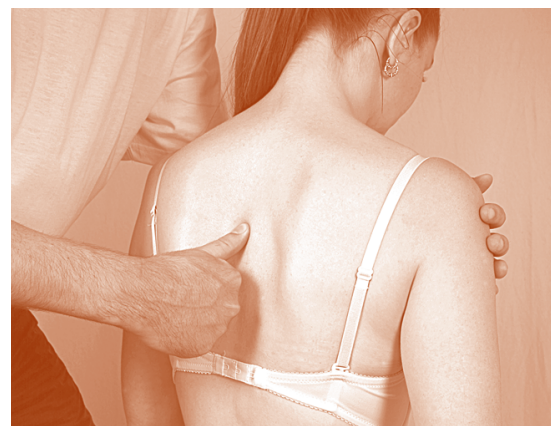

Abb. 2 Behandlung der Brustwirbelsäule. Quelle: siehe [1]

den Fingern zu umgreifen. Er wiederholt diesen Schritt einige Male, um die Muskulatur zu entspannen und merkt sich, wo an der Halswirbelsäule sich Muskelverhärtungen befinden.

Die Halswirbelsäule kann sowohl im Sitzen als auch im Liegen behandelt werden. Da Patienten die Behandlung im Liegen häufig als angenehmer empfinden, wird hier diese Variante erklärt.

\section{Behandlung der HWS im Liegen}

Die Patientin liegt mit leicht erhöht gelagerten Beinen auf dem Rücken. Der Kopf der Patientin liegt auf den Oberschenkeln des Therapeuten knapp oberhalb seiner Knie, sodass ihr Nacken gerade und entspannt ist ( Abb. 3). Der Therapeut sitzt am Kopfende der Behandlungsliege mit einem Handtuch auf dem Schoß. Seine Oberschenkel befinden sich auf der Höhe der Liege. Die Entfernung der Liege von den Knien beträgt $10-15 \mathrm{~cm}$. Zunächst massiert der Therapeut ein bis zwei Minuten von der Schulter aus in den Muskelrinnen am Nacken bis zum ersten Halswirbel hoch. Dazu setzt er die kleinen Finger oder Ringfinger beider Hände rechts und links neben der Halswirbelsäule an und zieht sie durch die Muskelrinnen bis zum Atlas hoch. Mittel- und Zeigefinger begleiten die Bewegung. Am Atlas angekommen, löst er zunächst den kleinen Finger, dann den Ringfinger, den Mittelfinger und schließlich den Zeigefinger vom Nacken. Durch diese Massage entspannt sich die Halsmuskulatur besonders gut, sodass die blockierten Wirbel anschließend gut zu tasten sind. Zur Korrektur eines blockierten Wirbels drückt er mit dem Mittelfinger auf die entsprechende Muskelverhärtung, während er den Kopf der Patientin mit beiden Hän-

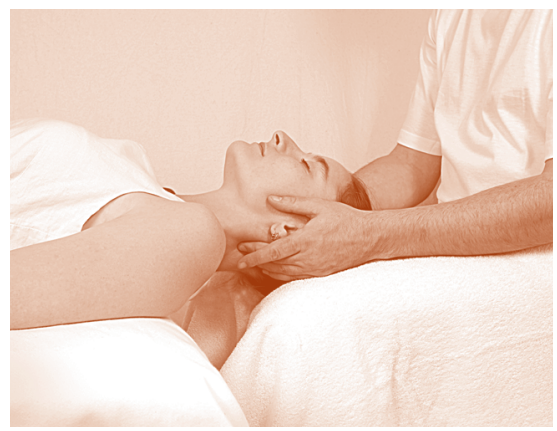

Abb. 3 Behandlung der Halswirbelsäule im Liegen. Quelle: siehe [1]

den rasch in kleinen, angedeuteten „nein“-Bewegungen abwechselnd nach links und rechts dreht. Der Bewegungsausschlag sollte dabei sehr gering bleiben und eher einer Vibration ähneln. Korrektur und Massage sollten ungefähr im Minutentakt abwechseln. Diese Behandlung sollte ungefähr eine Viertelstunde dauern.

Der Therapeut muss während der Behandlung darauf achten, der liegenden Patientin nicht die Ohren mit seinen Handflächen zuzuhalten, weil sie dies als unangenehm empfinden könnte.

\section{Mögliche Reaktionen auf die Behandlung}

Die meisten Patienten spüren keinerlei „Nebenwirkungen“, aber auf zwei Reaktionen möchten wir kurz hinweisen:

- Bei lang bestehenden Wirbelblockaden und -fehlstellungen kann nach der Behandlung Muskelkater auftreten. Er verweist darauf, dass sich die Fehlhaltungen ausgleichen und bedarf keiner besonderen Behandlung. Nach ein bis zwei Tagen gewöhnt sich die Muskulatur an die „neue“, richtige Belastung.

- Die wiederholten Streichungen der Behandlung der Halswirbelsäule können zu einer kurzzeitigen energetischen Fülle im Kopf mit Symptomen wie Schwindel oder Kopfweh führen. Diese sind harmlos und klingen rascher $a b$, wenn der Therapeut einige Male ohne Druck von oben nach unten über den Nacken streicht und die Patientin ein Glas Wasser trinkt.

+++ Bezugsquelle für Dorn-Bedarf,

Therapiebedarf Panek, Mindelheimstraße

51, 87666 Pforzen, Tel.: 08346/ 982356 ,

Fax: 08346/ 9823 68, http://www.therapiebedarf.net +++

\section{Literatur}

[1] Breidenbach O, Ewert R Lehrbuch Dorn-Therapie. Mit Breuß-Massage. 1. Aufl. Stuttgart: Sonntag; 2006

\section{Ausbildungsmöglichkeiten}

Naturheilpraxis Brigitte und Harald Fleig, Postfach $1232 \cdot 79657$ Wehr Tel.: 07762/72 60, Fax: 07762/35 67 E-Mail: harald.fleig@t-online.de

Gesundheits-Gemeinschaft Antje Kordts und Wolfgang Iwanowski Lewackerstraße 271 . 44798 Bochum Tel.: 0234/9490717

www.gesundheitsgemeinschaft.de

Dorn-Therapiezentrum Olaf Breidenbach, Piusstraße $127 \cdot 50931$ Köln

Tel.: 0221/99 25 727, Fax: 02 21/99 25729

www.dorn-therapiezentrum.de

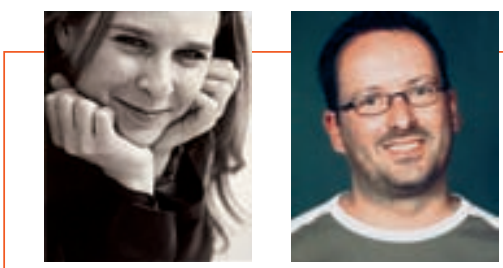

HP Rebecca Ewert

64 Hapua St, Hataitai,

Wellington, Neuseeland

E-Mail: rebecca@japanese-acupuncture. co.nz

\section{HP Olaf Breidenbach}

Piusstraße $127 \cdot 50931$ Köln

E-Mail: info@dorn-therapiezentrum.de

Rebecca Ewert ist Heilpraktikerin und arbeitet in eigener Praxis in Wellington, Neuseeland. Schwerpunktmäßig verwendet sie Klassische und Japanische Akupunktur und sanfte manuelle Techniken. Neben ihrer Praxistätigkeit schreibt und übersetzt sie Artikel und Vorträge über chinesische Medizin.

Olaf Breidenbach ist Leiter des DornTherapiezentrums in Köln. Seit 1996 arbeitet er intensiv mit der Dorn-Methode und gibt sie seit 2003 in eigenen Kursen weiter. In seiner täglichen Praxis kombiniert er die Dorn-Methode mit Traditioneller Chinesischer Medizin. 